\title{
Energy-Efficient Resource Allocation in CoMP-SWIPT Heterogeneous Networks
}

DOI:

10.1109/ICCChina.2017.8330360

\section{Document Version}

Accepted author manuscript

Link to publication record in Manchester Research Explorer

\section{Citation for published version (APA):}

Tang, J., So, D. K. C., Shojaeifard, A., \& Wong, K-K. (2017). Energy-Efficient Resource Allocation in CoMP-SWIPT Heterogeneous Networks. I E E E Transactions on Communications.

https://doi.org/10.1109/ICCChina.2017.8330360

\section{Published in:}

I E E E Transactions on Communications

\section{Citing this paper}

Please note that where the full-text provided on Manchester Research Explorer is the Author Accepted Manuscript or Proof version this may differ from the final Published version. If citing, it is advised that you check and use the publisher's definitive version.

\section{General rights}

Copyright and moral rights for the publications made accessible in the Research Explorer are retained by the authors and/or other copyright owners and it is a condition of accessing publications that users recognise and abide by the legal requirements associated with these rights.

\section{Takedown policy}

If you believe that this document breaches copyright please refer to the University of Manchester's Takedown Procedures [http://man.ac.uk/04Y6Bo] or contact uml.scholarlycommunications@manchester.ac.uk providing relevant details, so we can investigate your claim.

\section{OPEN ACCESS}




\title{
Energy-Efficient Resource Allocation in CoMP-SWIPT Heterogeneous Networks
}

\author{
Jie Tang ${ }^{1,2}$, Daniel K. C. So ${ }^{3}$, Arman Shojaeifard ${ }^{4}$ and Kai-Kit Wong ${ }^{4}$ \\ ${ }^{1}$ South China University of Technology, Email: eejtang@scut.edu.cn \\ ${ }^{2}$ The State Key Laboratory of Integrated Services Networks, Xidian University \\ ${ }^{3}$ University of Manchester, Email: d.so@manchester.ac.uk \\ ${ }^{4}$ University College London, Email: \{a.shojaeifard, kai-kit.wong\}@ucl.ac.uk.
}

\begin{abstract}
In this paper, a fundamental study of energy efficiency (EE) optimization for coordinated multi-point (CoMP)simultaneous wireless information and power transfer (SWIPT) heterogeneous networks (HetNets) is provided. We aim to optimize the EE whilst satisfying certain quality-of-service $(\mathrm{QoS})$ requirements in regard to transmission rate and energy harvesting at both the macro-cell and small-cells. The corresponding joint beamforming and power allocation in the presence of intra- and inter-cell interference constitutes a EE maximization problem that is non-convex, and hence very challenging to solve. In order to solve this problem, we propose to separate the beamforming design and power allocation processes. In particular, different from the the conventional linear zero-forcing beamforming where the beneficial interference is removed, a partial zero-forcing approach has been proposed by differentiating the energy harvesting users and information decoding users in order to improve the EE. Our findings show that the EE can be significantly improved through the integration of CoMP-SWIPT in HetNets.
\end{abstract}

\section{INTRODUCTION}

The rapid rise in traffic demands has driven the incentive for the research and development of the next generation of mobile networks, known as 5G. A key enabler for $5 \mathrm{G}$ is heterogeneous network (HetNet), where various types of base stations (BSs) are deployed to support user equipments (UEs) with increased density and quality-of-service (QoS) requirements [1]. On the other hand, the increased interference introduced through network densification in HetNets can limit the achievable spectral efficiency (SE). A prominent strategy for tackling the issue of interference is through cooperation, for example, using coordinated multi-point (CoMP) transmission [2]. Further, with the anticipated novel overhauls in the traditional cellular architecture such to limit the data exchange between various transmitters, CoMP techniques may be significantly more suited for deployment in $5 \mathrm{G}$ networks than the existing $4 \mathrm{G}$ systems. As a result, CoMP-enabled HetNet can be viewed as a prominent enabling technology for $5 \mathrm{G}$ and beyond [3].

Energy harvesting $(\mathrm{EH})$, on the other hand, is widely considered a de facto solution towards enhancing the lifespan of energy-constrained wireless devices such as low-power sensors [4]. Based on that, a new technology named wireless power transfer (WPT) enables the transmitter to transfer energy to the receivers with the help of radio frequency (RF) signals [5]. As a result, integrating RF-based EH capability in traditional wireless communication systems has emerged recently as an important research direction, namely, simultaneous wireless information and power transfer (SWIPT), where information decoding (ID) and EH are performed simultaneously [6].

As a result of the high energy requirements of wireless devices, energy efficiency (EE) performance has become a central theme towards delivering on the requirements of $5 \mathrm{G}$ in terms of user experience. With the introduction of the fundamental studies on SWIPT, EE maximization has attracted great attention in the context of SWIPT recently [7], [8]. Although the above studies are beneficial for understanding the theoretical bounds of SWIPT systems, there are certain challenges when it comes to implementing SWIPT technology in practice. For instance, power requirements/sensitivities for wireless information receivers and energy receivers are different and hence they are designed separately. This motivates our work to study the EE optimization for CoMP-SWIPT HetNet in a practical setting, where the BSs with multiple antennas simultaneously transmit to multiple single-antenna receivers with either information or energy, but not both at the same time. Under this setup, we consider joint beamforming and power allocation for QoS-constrained EE optimization in the downlink of CoMP-SWIPT two-tier HetNets, where minimum rate constraints for ID-UEs and minimum harvested energy constraints for EH-UEs are taken into consideration.

\section{System Model And Problem Formulation}

We consider the downlink of a CoMP-SWIPT two-tier HetNet comprising of a macro-cell and $L$ small-cells, as shown in Fig. 1. It is assumed that only one BS is providing service in each cell. The set of cells is denoted using $\mathcal{L}=\{0,1,2, \cdots, L\}$, where indexes 0 and $\{1,2, \cdots, L\}$ correspond to the macro-cell and the small-cells, respectively. For the SWIPT setup, two types of UEs are considered. In particular, the UEs are classified into ID UEs which receive and decode data from the corresponding BS, and EH UEs which are capable of transferring energy to the receivers using RF signals. $\mathcal{K}_{l}^{I}$ and $\mathcal{K}_{l}^{E}$ respectively represent the set of IDUEs connected to cell $l \in \mathcal{L}$ and the set of EH-UEs associated with cell $l \in \mathcal{L}$, and $\left|\mathcal{K}_{l}^{I}\right|=K_{l}^{I}$ and $\left|\mathcal{K}_{l}^{E}\right|=K_{l}^{E}$. The BS in cell $l \in \mathcal{L}$ is equipped with $N_{l}$ transmit antennas whilst 


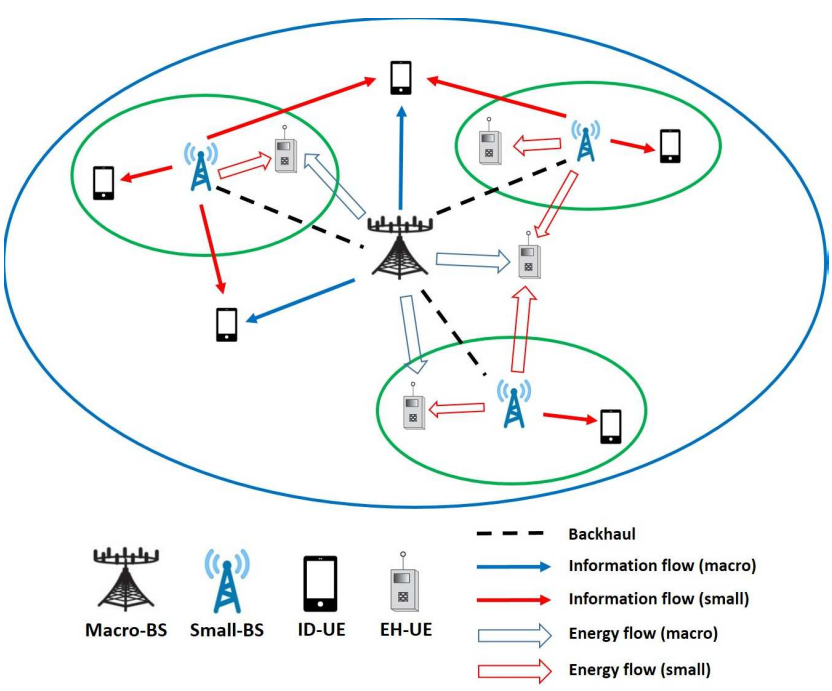

Fig. 1: A CoMP-SWIPT two-tier HetNet comprising of three smallcells and a macro-cell.

a single antenna is employed for all ID-UEs and EH-UEs. CoMP joint processing (CoMP-JP) and CoMP coordinated beamforming (CoMP-CB) are two different approaches that can provide high $\mathrm{SE}$ in downlink HetNets. In this paper, to emphasize the impact of heterogeneous deployment with SWIPT capabilities, the backhaul between the macro-BS and the small-BSs is assumed to be capacity-sufficient, and hence, CoMP-JP is employed. It is also assumed that perfect channel state information (CSI) is available at the BSs. For brevity, CoMP-JP is referred to as CoMP in the rest of the paper. Therefore, the transmitted signals at the BS $l$ is given by

$$
\mathbf{x}_{l}=\sum_{l \in \mathcal{L}} \sum_{k \in \mathcal{K}_{l}^{I}} \mathbf{w}_{[k, l]} s_{[k, l]}^{I}+\sum_{l \in \mathcal{L}} \sum_{k \in \mathcal{K}_{l}^{E}} \mathbf{v}_{[k, l]} s_{[k, l]}^{E},
$$

where $s_{[k, l]}^{I}$ and $\mathbf{w}_{[k, l]}$ respectively denote the data signal and its corresponding linear beamforming vector, $s_{[k, l]}^{E}$ and $\mathbf{v}_{[k, l]}$ are respectively representing the energy-carrying signal of EH$\mathrm{UE}[k, l]$ and its corresponding linear beamforming vector. In particular, we assume the data signal $s_{[k, l]}^{I}$ is independent and identically distributed (i.i.d) circularly symmetric complex Gaussian (CSCG) random variable with zero mean and unit variance. On the other hand, given that the energy signals carry no information, they can be modeled as random signals which meet certain requirements, i.e., power spectral density. Therefore, $s_{[k, l]}^{E}$ is assumed to be an independent white sequence with $\mathbb{E}\left[\left|s_{[k, l]}^{E}\right|^{2}\right]=1$. Furthermore, the transmit beamforming vectors are written as

$$
\begin{aligned}
\mathbf{w}_{[k, l]} & =\sqrt{p_{[k, l]}} \overline{\mathbf{w}}_{[k, l]}, \\
\mathbf{v}_{[k, l]} & =\sqrt{q_{[k, l]}} \overline{\mathbf{v}}_{[k, l]},
\end{aligned}
$$

where $p_{[k, l]}$ and $q_{[k, l]}$ are respectively denote the transmit power corresponding to the linear beamforming vector $\overline{\mathbf{w}}_{[k, l]}$ and $\overline{\mathbf{v}}_{[k, l]}$, and $\mathbb{E}\left[\left\|\overline{\mathbf{w}}_{[k, l]}\right\|^{2}\right]=\mathbb{E}\left[\| \overline{\mathbf{v}}_{[k, l]}||^{2}\right]=1$. The channel from the cell- $m$ BS to the $k$-th ID-UE in cell- $l$ is denoted as $\mathbf{h}_{[k, l]}^{m}$. Similarly, the channel from the cell- $m$ BS to the cell- $l$ $k$-th EH-UE is denoted with $\mathbf{g}_{[k, l]}^{m}$. Hence, suppose each IDUE treats the unintended signal data signals and all energy signals as noise, the interference received by ID-UE $[k, l]$ is

$$
\begin{gathered}
I_{[k, l]}=\sum_{j \in \mathcal{K}_{l}^{I} \backslash\{k\}}\left|\mathbf{w}_{[j, l]}^{H} \mathbf{h}_{[k, l]}^{l}\right|^{2}+\sum_{i \in \mathcal{L} \backslash\{l\}} \sum_{j \in \mathcal{K}_{i}^{I}}\left|\mathbf{w}_{[j, i]}^{H} \mathbf{h}_{[k, l]}^{i}\right|^{2} \\
+\sum_{i \in \mathcal{L}} \sum_{j \in \mathcal{K}_{i}^{E}}\left|\mathbf{v}_{[j, i]}^{H} \mathbf{h}_{[k, l]}^{i}\right|^{2} .
\end{gathered}
$$

Thus, the signal-to-interference-plus-noise ratio (SINR) at the ID-UE $[k, l]$ is given by

$$
\gamma_{[k, l]}=\frac{\left|\mathbf{w}_{[k, l]}^{H} \mathbf{h}_{[k, l]}^{l}\right|^{2}}{I_{[k, l]}+\sigma_{[k, l]}^{2}},
$$

where $\sigma_{[k, l]}^{2}$ is the noise power at the $k$-th ID-UE in cell- $l$. The transmission rate for the $k$-th ID-UE in cell- $l$ is given by

$$
R_{[k, l]}=W \log _{2}\left(1+\gamma_{[k, l]}\right),
$$

where $W$ denotes the total available spectrum.

The receiver with EH capability can harvest the energy from both data beams and energy beams, i.e., both w's and v's. Hence, the energy collected at the receiver of EH-UE $[k, l]$ is given by

$$
E_{[k, l]}=\eta\left(\sum_{i \in \mathcal{L}} \sum_{j \in \mathcal{K}_{i}^{I}}\left|\mathbf{w}_{[j, i]}^{H} \mathbf{g}_{[k, l]}^{i}\right|^{2}+\sum_{i \in \mathcal{L}} \sum_{j \in \mathcal{K}_{i}^{E}}\left|\mathbf{v}_{[j, i]}^{H} \mathbf{g}_{[k, l]}^{i}\right|^{2}\right),
$$

where $\eta$ indicates the loss when converting the energy.

For the considered CoMP-SWIPT HetNet system, the system's overall power consumption model should take into account the EH devices. Thus, similar to the power model used in [7], we consider the impact of harvested power, and hence, the system total power consumption is given by

$$
P=\zeta P_{T}+P_{C}-E,
$$

where $\zeta$ accounts for the reciprocal of the power amplifier drain efficiency, $P_{T}=\sum_{l \in \mathcal{L}} \sum_{k \in \mathcal{K}_{l}^{I}} p_{[k, l]}+$ $\sum_{l \in \mathcal{L}} \sum_{k \in \mathcal{K}_{l}^{E}} q_{[k, l]}$ is the transmission power, $E=$ $\sum_{l \in \mathcal{L}} \sum_{k \in \mathcal{K}_{l}^{E}} E_{[k, l]}$ is the harvested power at all EH-UEs. It should be noted that the minus sign in (8) indicates that the receivers are capable of harvesting energy that is radiated in the RF from the transmitter (a portion of the power). In addition, the circuit power consumption $P_{C}$ in our CoMPSWIPT HetNet can be modelled as,

$$
P_{C}=\sum_{l \in \mathcal{L}}\left(P_{a n t} N_{l}+P_{l}^{f i x}+P_{I} K_{l}^{I}+P_{E} K_{l}^{E}\right),
$$

where $P_{a n t} N_{l}$ represents the circuit power which depends on the number of transmit antennas at BS $l, P_{l}^{f i x}$ is the constant signal processing power at the $l^{\text {th }}$ cell. The last two terms $P_{I} K_{l}^{I}$ and $P_{E} K_{l}^{E}$ respectively denote the power required by all circuit components of each ID-UE and EH-UE.

The EE of our CoMP-SWIPT two-tier HetNet in the downlink can be formulated as (10) in the next page. Here, we are concerned with the problem of achieving maximum EE whilst meeting the required QoS constraints. Hence, the EE maximization problem should be formulated considering minimum transmission rate targets, minimum transferred en- 


$$
\lambda_{E E} \triangleq \frac{C}{P}=\frac{\sum_{l \in \mathcal{L}} \sum_{k \in \mathcal{K}_{l}^{I}} R_{[k, l]}}{\sum_{l \in \mathcal{L}}\left[\zeta\left(\sum_{k \in \mathcal{K}_{l}^{I}}\left|\mathbf{w}_{[k, l]}\right|^{2}+\sum_{k \in \mathcal{K}_{l}^{E}}\left|\mathbf{v}_{[k, l]}\right|^{2}\right)+P_{C}-\sum_{k \in \mathcal{K}_{l}^{E}} E_{[k, l]}\right]}
$$

ergy demands, and total power budgets. Accordingly, the EE problem for the CoMP-SWIPT HetNet is described as

$$
\begin{array}{ll}
\max _{\mathbf{w}_{[k, l]}, \mathbf{v}_{[k, l]}} & \lambda_{E E} \\
\text { s.t. } & \sum_{k \in \mathcal{K}_{l}^{I}}\left|\mathbf{w}_{[k, l]}\right|^{2}+\sum_{k \in \mathcal{K}_{l}^{E}}\left|\mathbf{v}_{[k, l]}\right|^{2} \leq P_{\text {max }}^{[l]}, \quad \forall l \in \mathcal{L}, \\
& R_{[k, l]} \geq \delta_{[k, l]}, \quad \forall\{k, l\} \in\left\{\mathcal{K}_{l}^{I}, \mathcal{L}\right\}, \\
& E_{[k, l]} \geq \rho_{[k, l]}, \quad \forall\{k, l\} \in\left\{\mathcal{K}_{l}^{E}, \mathcal{L}\right\},
\end{array}
$$

where $P_{\max }^{[l]}$ represents the transmit power budget for the cell$l, \delta_{[k, l]}$ represents the minimum rate target of ID-UE $[k, l]$ and $\rho_{[k, l]}$ denotes the minimum harvested energy for EH-UE $[k, l]$. Therefore, constraints (12)-(14) are respectively used to guarantee the maximum power budget, the minimum rate target and the minimum harvested energy requirement.

The EE optimization problem, with joint beamforming and power allocation in the presence of inter- and intra-cell interference, is mixed-combinatorial and non-convex. The solution is therefore non-trivial and cannot be obtained directly. In order to solve this EE optimization problem, one may rely on an exhaustive search method over all the possible beamforming and power allocation combinations. Nevertheless, it is obvious that this exhaustive search method incurs intensive computational complexity in the number of transmit antennas, UEs, and small-cells. As a result, a low complexity resource allocation strategy is developed with the help of ZF beamforming.

\section{PZF-BASEd Resource Allocation Scheme}

In this section, we introduce the beamforming design and power allocation strategy for the proposed CoMP-SWIPT HetNet. Since the original problem in (11)-(14) is non-convex, it is non-trivial to derive the optimal beamforming directly, i.e., optimal $\mathbf{w}$ and $\mathbf{v}$. Even if we can develop an optimal joint beamforming and power allocation strategy, the computational complexity is often too high when considering a practical HetNet scenario where there exists densely-populated smallcells and UEs. Thus, we separate the beamforming and power allocation to facilitate possible implementation in practice.

The most common precoding and receive combining that are used in current wireless communication networks are $\mathrm{ZF}$, maximum ratio transmission/combining (MRT/MRC), and minimum mean squared error (MMSE) processing. However, it has been shown in [9] that ZF precoding provides the highest EE in a CoMP-enabled HetNet. However, the idea of the ZFbased solution is to remove the intra- and inter-cell interference to both ID-UEs and EH-UEs, and hence it is efficient for data transmission (Fig. 2). It is true that the interference is a degrading factor for all ID-UEs. However, interference is beneficial to all EH-UEs since they can collect energy from the surrounding environment. Therefore, to design the beamforming that is suitable for our CoMP-SWIPT HetNet scenario, we can differentiate between the EH-UEs and ID-UEs due to their different performance with respect to interference.

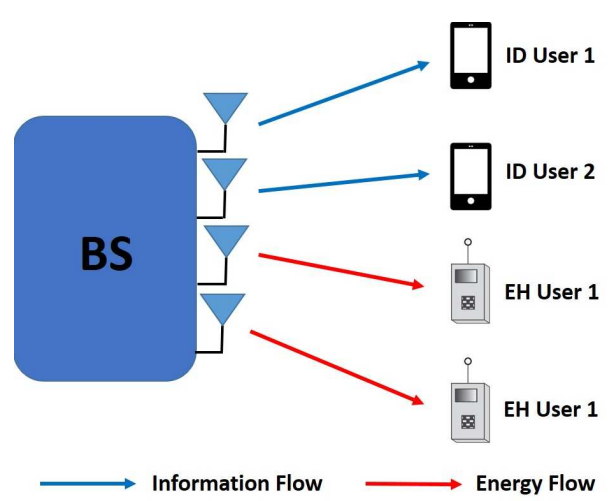

Fig. 2: Illustration of the signal space in CoMP-SWIPT HetNet with the $\mathrm{ZF}$ approach.

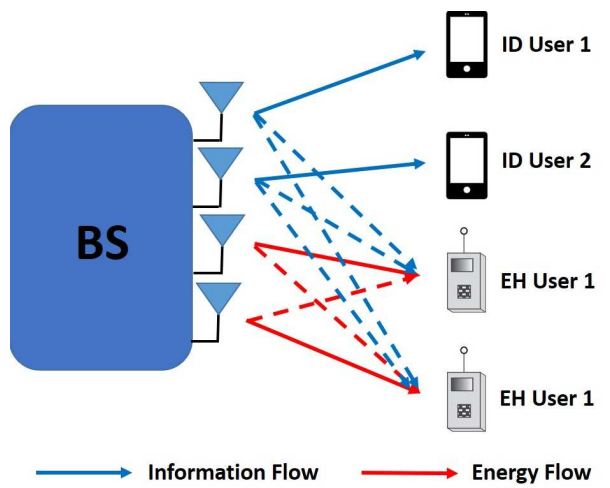

Fig. 3: Illustration of the signal space in CoMP-SWIPT HetNet with the PZF approach.

To fully exploit all existing interference, the ideal interference control strategy of the proposed CoMP-SWIPT is shown in Fig. 3. In particular, the precoder for the ID-UEs, $\overline{\mathbf{w}}_{[k, l]}, \forall\{k, l\} \in\left\{\mathcal{K}_{l}^{I}, \mathcal{L}\right\}$, can be designed based on ZF beamforming strategy. On the other hand, we devise the EH precoder $\overline{\mathbf{v}}_{[k, l]}, \forall\{k, l\} \in\left\{\mathcal{K}_{l}^{E}, \mathcal{L}\right\}$ with the fact that the interference is beneficial for all EH-UEs should be considered. Therefore, on the basis of interference impact of ID-UEs and EH-UEs, the design of the precoder for ID-UEs and EH-UEs should be perform separately.

We first construct the null space for ID-UE $\left[k^{*}, l^{*}\right]$ as

$\overline{\mathbf{N}}_{\left[k^{*}, l^{*}\right]}^{I} \subset \operatorname{null}\left(\left[\left(\mathbf{h}_{\left[k\left(k \in \mathcal{K}_{l}^{I}\right), l\left(l \in \mathcal{L} \backslash\left\{l^{*}\right\}\right)\right]}^{l^{*}}\right)\left(\mathbf{h}_{\left[k\left(k \in \mathcal{K}_{l^{*}}^{I} \backslash\left\{k^{*}\right\}\right), l^{*}\right]}^{l^{*}}\right){ }^{H}\right)\right.$.

Based on the constructed null space, the hybrid beamforming for ID-UE $\left[k^{*}, l^{*}\right]$, which consists of a PZF precoder, is written as

$$
\mathbf{w}_{\left[k^{*}, l^{*}\right]}=\overline{\mathbf{N}}_{\left[k^{*}, l^{*}\right]}^{I} \tilde{\mathbf{w}}_{\left[k^{*}, l^{*}\right]},
$$

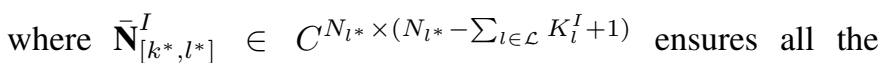
interference for ID-UEs is removed, and $\tilde{\mathbf{w}}_{\left[k^{*}, l^{*}\right]} \in$ $C^{\left(N_{l^{*}}-\sum_{l \in \mathcal{L}} K_{l}^{I}+1\right) \times 1}$ is used to exploit the column space of $\overline{\mathbf{N}}_{\left[k^{*}, l^{*}\right]}^{I}$ in order to further improve EE. In other words, the 
hybrid transmit beamforming $\mathbf{w}_{\left[k^{*}, l^{*}\right]}$ is capable of cancelling interference to ID-UEs whilst maximizing EE.

On the other hand, to fully exploit the interference, we construct the null space for EH-UEs in the cell $\left[l^{*}\right]$ as follows,

$$
\overline{\mathbf{N}}_{\left[l^{*}\right]}^{E} \subset \operatorname{null}\left(\left[\mathbf{h}_{\left[k\left(k \in \mathcal{K}_{l}^{I}\right), l(l \in \mathcal{L})\right]}^{l^{*}}\right]^{H}\right),
$$

such that the energy-carrying signal transmitted from BS $l^{*}$ do not generate interference to all the ID-UEs. It should be noted that these null spaces for EH-UEs are shared within the cell, i.e., $\overline{\mathbf{N}}_{\left[l^{*}\right]}^{E}=\overline{\mathbf{N}}_{\left[1, l^{*}\right]}^{E}=\cdots=\overline{\mathbf{N}}_{\left[K_{L}^{E}, l^{*}\right]}^{E}$. Hence, we can write the hybrid beamforming for $\mathrm{EH}-\mathrm{UE}\left[k^{*}, l^{*}\right]$ based on the above constructed null space, which is given by

$$
\mathbf{v}_{\left[k^{*}, l^{*}\right]}=\overline{\mathbf{N}}_{\left[l^{*}\right]}^{E} \tilde{\mathbf{v}}_{\left[k^{*}, l^{*}\right]},
$$

where $\overline{\mathbf{N}}_{\left[l^{*}\right]}^{E} \in C^{N_{l^{*}} \times\left(N_{l^{*}}-\sum_{l \in \mathcal{L}} K_{l}^{I}\right)}$ represents the spaces that are shared by all the EH-UEs in the cell $l^{*}$. Similarly, $\tilde{\mathbf{v}}_{\left[k^{*}, l^{*}\right]} \in$ $C^{\left(N_{l^{*}}-\sum_{l \in \mathcal{L}} K_{l}^{I}\right) \times 1}$ is a vector that combines the columns of $\overline{\mathbf{N}}_{\left[l^{*}\right]}^{E}$ towards optimizing the EE. The effective channels are then given by

$$
\begin{gathered}
a_{[k, l]}^{\left[k^{*}, l^{*}\right]}=\overline{\mathbf{N}}_{\left[k^{*}, l^{*}\right]}^{I H} \mathbf{h}_{[k, l]}^{l^{*}}, \\
b_{[k, l]}^{\left[k^{*}, l^{*}\right]}=\overline{\mathbf{N}}_{\left[k^{*}, l^{*}\right]}^{I H} \mathbf{g}_{[k, l]}^{l^{*}}, \\
c_{[k, l]}^{\left[k^{*}, l^{*}\right]}=\overline{\mathbf{N}}_{\left[l^{*}\right]}^{E H} \mathbf{g}_{[k, l]}^{l^{*}},
\end{gathered}
$$

where $a_{[k, l]}^{\left[k^{*}, l^{*}\right]}$ represents the effective channel from BS $l^{*}$ to $\mathrm{ID}-\mathrm{UE}[k, l]$ with the PZF beamformer of ID-UE $\left[k^{*}, l^{*}\right]$, $b_{[k, l]}^{\left[k^{*}, l^{*}\right]}$ denotes the effective channel from BS $l *$ to EH-UE $[k, l]$ with the PZF beamformer of ID-UE $\left[k^{*}, l^{*}\right]$, and $c_{[k, l]}^{\left[k^{*}, l^{*}\right]}$ indicates the effective channel from $\mathrm{BS} l *$ to $\mathrm{EH}-\mathrm{UE}[k, l]$ with the PZF beamformer of EH-UE $\left[k^{*}, l^{*}\right]$.

Hence, with the proposed hybrid beamforming, our target is to design the effective beamformers $\tilde{\mathbf{w}}_{\left[k^{*}, l^{*}\right]}$ and $\tilde{\mathbf{v}}_{\left[k^{*}, l^{*}\right]}$ such that the system EE is maximized. To achieve this, the original optimization problem in (11)-(14) is reformulated as

$$
\begin{gathered}
\max _{\tilde{\mathbf{w}}_{[k, l]} \tilde{\mathbf{v}}_{[k, l]}} \frac{\sum_{l \in \mathcal{L}} \sum_{k \in \mathcal{K}_{l}^{I}} W \log _{2}\left(1+\frac{\left|a_{[k, l]}^{[k, l] H} \tilde{\mathbf{w}}_{[k, l]}\right|^{2}}{\sigma_{[k, l]}^{2}}\right)}{\text { s.t. } \sum_{k \in \mathcal{K}_{l}^{I}}\left|\tilde{\mathbf{w}}_{[k, l]}\right|^{2}+\sum_{k \in \mathcal{K}_{l}^{E}}\left|\tilde{\mathbf{w}}_{[v, l]}\right|^{2} \leq P_{m a x}^{[l]}, \forall l \in \mathcal{L},} \\
\\
W \log _{2}\left(1+\frac{\left|a_{[k, l]}^{[k, l] H} \tilde{\mathbf{w}}_{[k, l]}\right|^{2}}{\sigma_{[k, l]}^{2}}\right) \geq \delta_{[k, l]}, \forall\{k, l\} \in\left\{\mathcal{K}_{l}^{I}, \mathcal{L}\right\}, \\
\\
\quad \sum_{l * \in \mathcal{L}} \sum_{k^{*} \in \mathcal{K}_{l}^{I}}\left|b_{[k, l]}^{\left[k^{*}, l^{*}\right] H} \tilde{\mathbf{w}}_{\left[k^{*}, l^{*}\right]}\right|^{2} \\
\left.+\sum_{l^{*} \in \mathcal{L}} \sum_{k^{*} \in \mathcal{K}_{l^{*}}^{E}}\left|c_{[k, l]}^{\left[k^{*}, l^{*}\right] H} \tilde{\mathbf{v}}_{\left[k^{*}, l^{*}\right]}\right|^{2}\right) \geq \rho_{[k, l]}, \forall\{k, l\} \in\left\{\mathcal{K}_{l}^{E}, \mathcal{L}\right\},
\end{gathered}
$$

where $\tilde{P}=\sum_{l \in \mathcal{L}}\left[\zeta\left(\sum_{k \in \mathcal{K}_{l}^{I}}\left|\tilde{\mathbf{w}}_{[k, l]}\right|^{2}+\sum_{k \in \mathcal{K}_{l}^{E}}\left|\tilde{\mathbf{v}}_{[k, l]}\right|^{2}\right)\right]+$ $P_{f i x}-\sum_{l \in \mathcal{L}} \sum_{k \in \mathcal{K}_{l}^{E}} E_{[k, l]}, P_{f i x}=\sum_{l \in \mathcal{L}}\left(P_{a n t} N_{l}+P_{l}^{s t a}\right)$. Unfortunately, the above optimization problem is a non-convex non-linear fractional programming problem. The solution is therefore nontrivial and cannot be obtained directly.
1) Initialize $\beta=0$ and the stopping criteria $\delta=1 e^{-3 \text {; }}$

2) REPEAT

3) Under a fixed $\beta$, tackle problem (26)-(29) to obtain the beamforming $\left\{\tilde{\mathbf{W}}_{[k, l]}, \tilde{\mathbf{V}}_{[k, l]}\right\}$;

4) $\quad$ IF $U_{R}\left(\tilde{\mathbf{W}}_{[k, l]}, \tilde{\mathbf{V}}_{[k, l]}\right)-\beta U_{T}\left(\tilde{\mathbf{W}}_{[k, l]}, \tilde{\mathbf{V}}_{[k, l]}\right) \leq \delta$

5) Convergence $=$ TRUE;

6) $\quad$ RETURN $\left\{\tilde{\mathbf{W}}_{[k, l]}^{*}, \tilde{\mathbf{V}}_{[k, l]}^{*}\right\}=\left\{\tilde{\mathbf{W}}_{[k, l]}, \tilde{\mathbf{V}}_{[k, l]}\right\}$ and $\beta^{*}=\frac{U_{R}\left(\tilde{\mathbf{W}}_{[k, l]}, \tilde{\mathbf{V}}_{[k, l]}\right)}{U_{T}\left(\tilde{\mathbf{W}}_{[k, l]}, \tilde{\mathbf{V}}_{[k, l]}\right)}$;

\section{7) ELSE}

8) $\quad$ Set $\beta=\frac{U_{R}\left(\tilde{\mathbf{W}}_{[k, l]}, \tilde{\mathbf{V}}_{[k, l]}\right)}{U_{T}\left(\tilde{\mathbf{W}}_{[k, l]}, \tilde{\mathbf{V}}_{[k, l]}\right)}$ and $n=n+1$,

Convergence = FALSE;

\section{9) END IF}

10) UNTIL Convergence $=$ TRUE.

TABLE I: Proposed Dinkelbach method-based solution.

To solve this problem, we first define the beamforming and the effective channels in matrix form, i.e., $\tilde{\mathbf{W}}_{[k, l]}=\tilde{\mathbf{w}}_{[k, l]} \tilde{\mathbf{w}}_{[k, l]}^{H}$, $\tilde{\mathbf{v}}_{[k, l]}=\tilde{\mathbf{v}}_{[k, l]} \tilde{\mathbf{v}}_{[k, l]}^{H}, \mathbf{A}_{[k, l]}^{\left[k^{*}, l^{*}\right]}=\frac{1}{\sigma_{[k, l]}^{2}} a_{[k, l]}^{\left[k^{*}, l^{*}\right]} a_{[k, l]}^{\left[k^{*}, l^{*}\right] H}, \mathbf{B}_{[k, l]}^{\left[k^{*}, l^{*}\right]}=$ $b_{[k, l]}^{\left[k^{*}, l^{*}\right]} b_{[k, l]}^{\left[k^{*}, l^{*}\right] H}, \quad \mathbf{C}_{[k, l]}^{\left[k^{*}, l^{*}\right]}=c_{[k, l]}^{\left[k^{*}, l^{*}\right]} c_{[k, l]}^{\left[k^{*}, l^{*}\right] H}$. In addition, it has been shown in [10] that the Dinkelbach method is an effective scheme to solve the classic non-convex non-linear fractional programming problems. Therefore, with the help of Dinkelbach method, the EE maximization problem in (22)-(25) can be solved by transforming the fractional-form function into a subtractive-form function that is summarized in Table I.

As detailed in Table I, the key of the proposed approach is to tackle the equivalent problem under a fixed $\beta$, i.e., step 3,

$$
\begin{aligned}
& \max _{\tilde{\mathbf{W}}_{[k, l]}, \tilde{\mathbf{V}}_{[k, l]}} \sum_{l \in \mathcal{L}} \sum_{k \in \mathcal{K}_{l}^{I}} W \log _{2}\left(1+\operatorname{tr}\left(\mathbf{A}_{[k, l]}^{[k, l]} \tilde{\mathbf{W}}_{[k, l]}\right)\right. \\
& -\beta\left\{\sum_{l \in \mathcal{L}}\left[\zeta\left(\sum_{k \in \mathcal{K}_{l}^{I}} \operatorname{tr}\left(\tilde{\mathbf{W}}_{[k, l]}\right)+\sum_{k \in \mathcal{K}_{l}^{E}} \operatorname{tr}\left(\tilde{\mathbf{V}}_{[k, l]}\right)\right)\right]\right. \\
& \left.+P_{f i x}-\sum_{l \in \mathcal{L}} \sum_{k \in \mathcal{K}_{l}^{E}} E_{[k, l]}\right\} \\
& \text { s.t. } \sum_{k \in \mathcal{K}_{l}^{I}} \operatorname{tr}\left(\tilde{\mathbf{W}}_{[k, l]}\right)+\sum_{k \in \mathcal{K}_{l}^{E}} \operatorname{tr}\left(\tilde{\mathbf{V}}_{[k, l]}\right) \leq P_{\max }^{[l]}, \forall l \in \mathcal{L}, \quad(27) \\
& \quad W \log _{2}\left(1+\operatorname{tr}\left(\mathbf{A}_{[k, l]}^{[k, l]} \tilde{\mathbf{W}}_{[k, l]}\right) \geq \delta_{[k, l]}, \forall\{k, l\} \in\left\{\mathcal{K}_{l}^{I}, \mathcal{L}\right\}\right. \\
& \quad \eta\left[\sum_{l^{*} \in \mathcal{L}} \sum_{k^{*} \in \mathcal{K}_{l}^{I}} \operatorname{tr}\left(\mathbf{B}_{[k, l]}^{\left[k^{*}, l^{*}\right]} \tilde{\mathbf{W}}_{\left[k^{*}, l^{*}\right]}\right)\right. \\
& +\sum_{l^{*} \in \mathcal{L}} \sum_{k^{*} \in \mathcal{K}_{l^{*}}^{E}} \operatorname{tr}\left(\mathbf{C}_{[k, l]}^{\left[k^{*}, l^{*}\right]} \tilde{\mathbf{V}}_{\left[k^{*}, l^{*}\right]}\right) \geq \rho_{[k, l]}, \forall\{k, l\} \in\left\{\mathcal{K}_{l}^{E}, \mathcal{L}\right\} .
\end{aligned}
$$

Since $\operatorname{tr}(\mathbf{X})$ is linear with $\mathbf{X}$, and $\log |\mathbf{X}|$ is a concave function [11], the optimization problem (26)-(29) is convex. As a result, similar to the method in [12], the optimal solution, i.e., $\tilde{\mathbf{W}}_{[k, l]}$ and $\tilde{\mathbf{V}}_{[k, l]}$, can be achieved with the help of the gradient approach. In particular, we update $\tilde{\mathbf{W}}_{[k, l]}$ and $\tilde{\mathbf{V}}_{[k, l]}$ consecutively as follows,

$$
\tilde{\mathbf{W}}_{[k, l]}(n)=\left[\tilde{\mathbf{W}}_{[k, l]}(n-1)+\varpi(n-1) \nabla_{\tilde{\mathbf{W}}_{[k, l]}} \tilde{G}\right]^{+},
$$




$$
\tilde{\mathbf{V}}_{[k, l]}(n)=\left[\tilde{\mathbf{V}}_{[k, l]}(n-1)+\bar{\varpi}(n-1) \nabla_{\tilde{\mathbf{V}}_{[k, l]}} \tilde{G}\right]^{+},
$$

where $\varpi$ and $\bar{\varpi}$ represent the step size, and $\tilde{G}$ is the corresponding Lagrangian function for problem (26)-(29) which is given by (32) in the next page, with the gradient as

$$
\begin{gathered}
\nabla_{\tilde{\mathbf{W}}_{[k, l]}} \tilde{G}=\frac{W\left(1+\tilde{\varrho}_{[k, l]}\right) \mathbf{A}_{[k, l]}^{[k, l]}}{1+\operatorname{tr}\left(\mathbf{A}_{[k, l]}^{[k, l]} \tilde{\mathbf{W}}_{[k, l]}\right)}-\left(\beta \zeta+\tilde{\xi}_{l}\right) \mathbf{I}+ \\
\left(\beta-\tilde{\kappa}_{[k, l]}\right) \eta \sum_{l^{*} \in \mathcal{L}} \sum_{k^{*} \in \mathcal{K}_{l}^{I}} \mathbf{B}_{\left[k^{*}, l^{*}\right]}^{[k, l]}, \\
\nabla_{\tilde{\mathbf{V}}_{[k, l]}} \tilde{G}=-\left(\beta \zeta+\tilde{\xi}_{l}\right) \mathbf{I}+\left(\beta-\tilde{\kappa}_{[k, l]}\right) \eta \sum_{l^{*} \in \mathcal{L}} \sum_{k^{*} \in \mathcal{K}_{l}^{E}} \mathbf{C}_{\left[k^{*}, l^{*}\right]}^{[k, l]}
\end{gathered}
$$

Once the optimal $\tilde{\mathbf{W}}_{[k, l]}$ and $\tilde{\mathbf{V}}_{[k, l]}$ are obtained using (30) and (31), the corresponding optimal Lagrangian dual variables $\tilde{\xi}_{l}, \tilde{\varrho}_{[k, l]}, \tilde{\kappa}_{[k, l]}$ can be determined. In particular, with the help of the sub-gradient method, the optimal Lagrangian dual variables can be updated as follows,

$$
\begin{gathered}
\xi_{l}(n)=\left[\xi_{l}(n-1)\right. \\
\left.+\tilde{\varpi}(n-1)\left(P_{m a x}^{[l]}-\sum_{k \in \mathcal{K}_{l}^{I}} \operatorname{tr}\left(\tilde{\mathbf{W}}_{[k, l]}\right)-\sum_{k \in \mathcal{K}_{l}^{E}} \operatorname{tr}\left(\tilde{\mathbf{V}}_{[k, l]}\right)\right)\right]^{+} \\
\varrho_{[k, l]}(n)=\left[\varrho_{[k, l]}(n-1)\right. \\
\left.+\hat{\varpi}(n-1)\left(W \log _{2}\left(1+\operatorname{tr}\left(\mathbf{A}_{[k, l]}^{[k, l]} \tilde{\mathbf{W}}_{[k, l]}\right)\right)-\delta_{[k, l]}\right)\right]^{+}, \\
\kappa_{[k, l]}(n)=\left[\kappa_{[k, l]}(n-1)+\check{\varpi}(n-1)\left(E_{[k, l]}-\rho_{[k, l]}\right)\right]^{+},
\end{gathered}
$$

and $\tilde{\varpi}, \hat{\varpi}$ and $\check{\varpi}$ represents the updating step size.

\section{Simulation Results}

In this section, simulation results are provided in order to validate the performance of the proposed PZF-based approach in the CoMP-SWIPT HetNet. It is assumed that three uniformly-distributed small-cells are in the coverage area of a macro-cell, where the radius of the macro-cell and the smallcells are set to $250 \mathrm{~m}$ and $50 \mathrm{~m}$, respectively, and there exists two ID-UEs and two EH-UEs in each cell. In order to model the capability of EH devices, all EH-UEs are considered to be located within $20 \mathrm{~m}$ to the corresponding BSs. We assume that the channels are i.i.d quasi-static Rayleigh block fading. Moreover, the path-loss parameter is set to 2.5 and 3.76 respectively for a practical line-of-sight SWIPT scenario and a typical 3GPP propagation environment. The power amplifier drain efficiency $\zeta$ and the EH efficiency $\eta$ are set to $30 \%$ and $10 \%$, respectively. In order to provide different service priorities and guaranteed QoS for each UE, the minimum rate constraint per ID-UE $\delta_{[k, l]}$ is set to $2 \mathrm{~b} / \mathrm{s} / \mathrm{Hz}$, and the minimum harvested energy per EH-UE $\rho_{[k, l]}$ is set to $0.2 \mathrm{~W}$.

First, we investigate the convergence behavior of the proposed PZF-based scheme. It can be seen from Fig. 4 that the proposed algorithm is successfully converging to the optimal solution with around 90 iterations. Furthermore, as expected, the proposed PZF based approach outperforms the ZF based scheme in terms of EE (approximately 11\%). This is

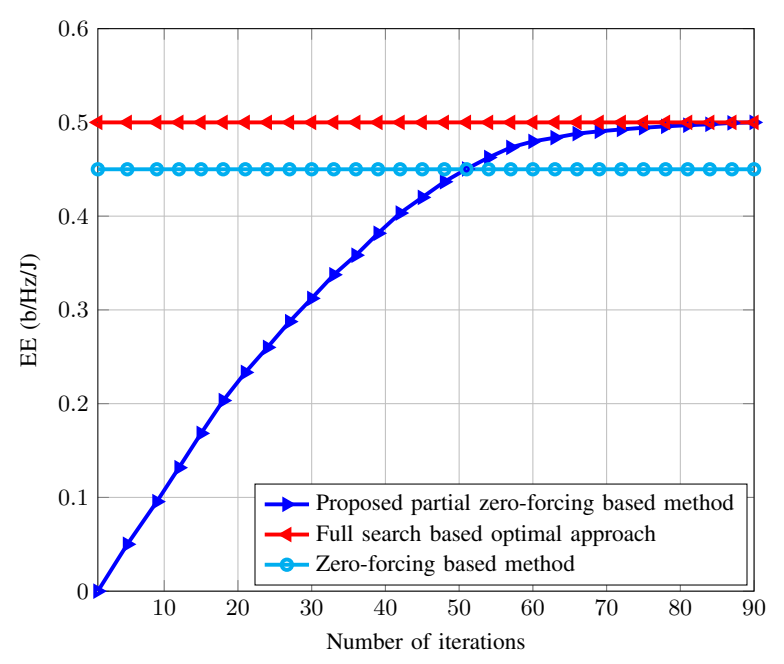

Fig. 4: Convergence behavior of the proposed PZF-based approach.

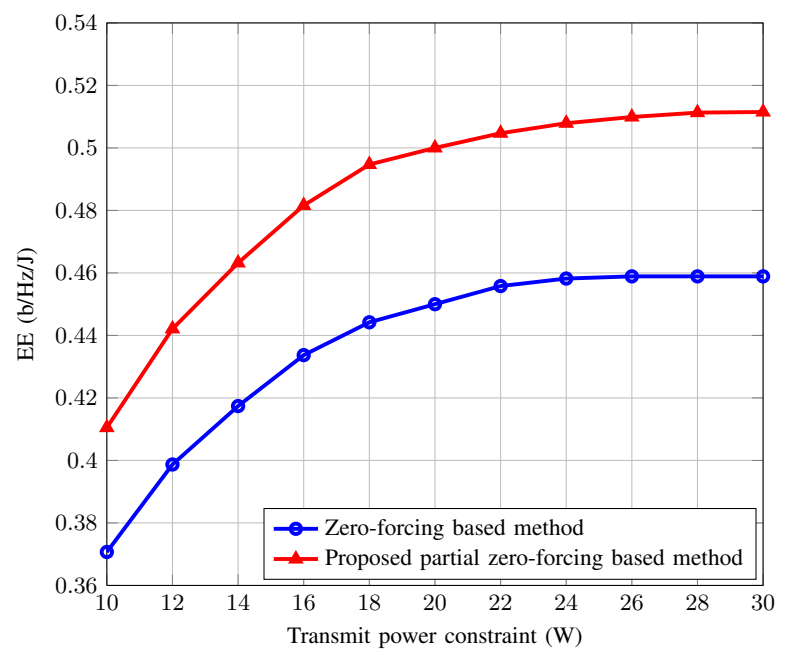

Fig. 5: Performance of the proposed PZF-based approach (EE vs transmit power constraint).

because the former approach aims to fully utilize the beneficial interference for $\mathrm{EH}$ purposes without weakening the quality of data transmission, and hence, a higher EE is achieved.

In the next simulation, the EE performance of the proposed PZF-based scheme with various QoS demands are evaluated. We first investigate the EE performance of the proposed solution with different power budgets. As shown in Fig. 5, the optimal EE achieved by the proposed PZF-based scheme is monotonically non-decreasing in the total transmit power constraint per cell $P_{\max }^{[l]}$. Particularly, the EE increases dramatically with a smaller power budget, i.e., $10<P_{\max }<24$ $\mathrm{W}$, and then approaches an asymptotic value where a balance among the achievable rates and the total power consumption is obtained. We next show in Fig. 6 that the optimal EE under different harvested energy requirement. We can observe that the maximum EE remains unchanged up to a particular minimum harvested energy constraint, but decreases thereafter. This result implies that the best energy-efficient approach is using a higher transmit power. However, to satisfy a higher minimum harvested energy demand, extra power is required. It has been shown in Fig. 5 that this additional power cannot 


$$
\begin{aligned}
& \tilde{G}\left(\tilde{\mathbf{W}}_{[k, l]}, \tilde{\mathbf{V}}_{[k, l]}, \tilde{\xi}_{l}, \tilde{\varrho}_{[k, l]}, \tilde{\kappa}_{[k, l]}\right)=\sum_{l \in \mathcal{L}} \sum_{k \in \mathcal{K}_{l}^{I}} W \log _{2}\left(1+\operatorname{tr}\left(\mathbf{A}_{[k, l]}^{[k, l]} \tilde{\mathbf{W}}_{[k, l]}\right)-\beta\left\{\sum_{l \in \mathcal{L}}\left[\zeta\left(\sum_{k \in \mathcal{K}_{l}^{I}} \operatorname{tr}\left(\tilde{\mathbf{W}}_{[k, l]}\right)+\sum_{k \in \mathcal{K}_{l}^{E}} \operatorname{tr}\left(\tilde{\mathbf{V}}_{[k, l]}\right)\right)\right]\right.\right. \\
& \left.+P_{f i x}-\sum_{l \in \mathcal{L}} \sum_{k \in \mathcal{K}_{l}^{E}} E_{[k, l]}\right\}+\sum_{l \in \mathcal{L}} \tilde{\xi}_{l}\left(P_{m a x}^{[l]}-\sum_{k \in \mathcal{K}_{l}^{I}} \operatorname{tr}\left(\tilde{\mathbf{W}}_{[k, l]}\right)-\sum_{k \in \mathcal{K}_{l}^{E}} \operatorname{tr}\left(\tilde{\mathbf{V}}_{[k, l]}\right)\right)+\sum_{l \in \mathcal{L}} \sum_{k \in \mathcal{K}_{l}^{I}} \tilde{\varrho}_{[k, l]}\left[W \log _{2}\left(1+\operatorname{tr}\left(\mathbf{A}_{[k, l]}^{[k, l]} \tilde{\mathbf{W}}_{[k, l]}\right)-\delta_{[k, l]}\right]\right. \\
& +\sum_{l \in \mathcal{L}} \sum_{k \in \mathcal{K}_{l}^{E}} \tilde{\kappa}_{[k, l]}\left\{\eta\left[\sum_{l^{*} \in \mathcal{L}} \sum_{k^{*} \in \mathcal{K}_{l}^{I}} \operatorname{tr}\left(\mathbf{B}_{[k, l]}^{\left[k^{*}, l^{*}\right]} \tilde{\mathbf{W}}_{\left[k^{*}, l^{*}\right]}\right)+\sum_{l^{*} \in \mathcal{L}} \sum_{k^{*} \in \mathcal{K}_{l^{*}}^{E}} \operatorname{tr}\left(\mathbf{C}_{[k, l]}^{\left[k^{*}, l^{*}\right]} \tilde{\mathbf{V}}_{\left[k^{*}, l^{*}\right]}\right)\right]-\rho_{[k, l]}\right\}
\end{aligned}
$$

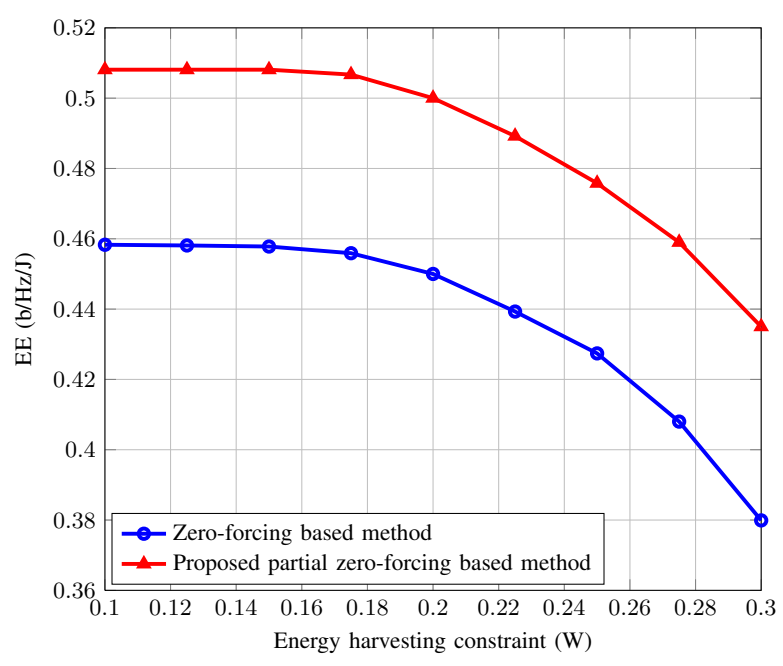

Fig. 6: Performance of the proposed PZF-based approach (EE vs EH constraint).

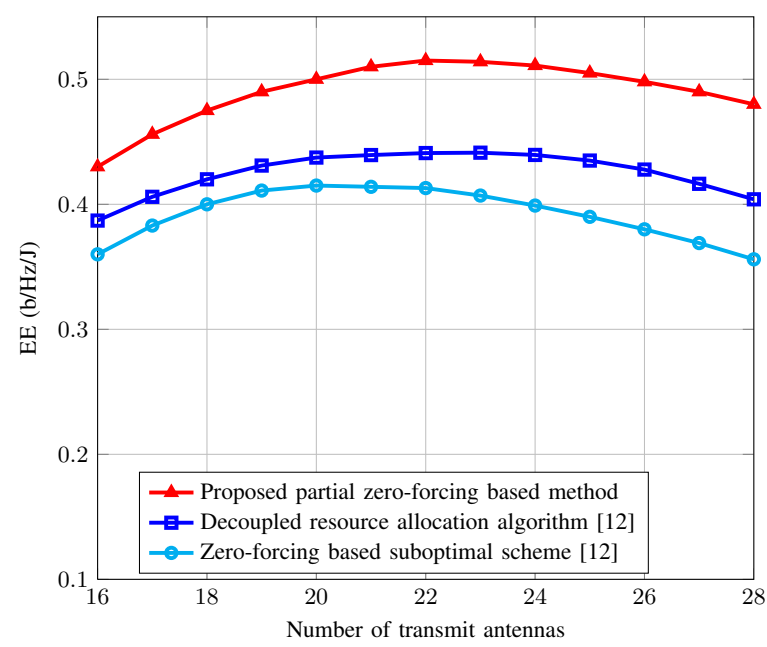

Fig. 7: Impact of the number of transmit antennas on the EE performance under different resource allocation approaches.

provide any EE gain, but in fact, it will weaken the EE performance due to the smaller transmit power range.

Finally, the effect of the number of transmit antennas on the EE in the CoMP-SWIPT HetNet has been studied. In order to depict the EE gain of the proposed scheme, we compare the performance with a approach that optimizes the EE in a conventional two-tier HetNet (without EH capability). In particular, we take into account the approach with joint transmit beamforming design and power allocation policy in [12], and the ZF based suboptimal scheme in [12]. As shown in Fig. 7, the proposed PZF-based solution obtains a superior EE versus all other approaches. Specifically, the EE first increases then decreases with the increasing number of transmit antennas, and the optimal value is 22 .

\section{CONCLusions}

In this paper, we studied the problem of QoS-constrained EE maximization in the downlink of CoMP-SWIPT HetNets. The corresponding optimization problem in the presence of interand intra-cell interference leads to a non-convex problem, and thus the solution is non-trivial and cannot be obtained directly. To solve this problem, a decoupled algorithm has been proposed where the beamforming design and power allocation procedure are separated. In particular, by utilizing the notion that interference benefits the EH-UEs, we propose a PZF approach by differentiating the EH-UEs and ID-UEs in order to improve the EE. Through constructing null spaces, a hybrid transmit beamforming was then developed to optimally combine the column spaces towards maximizing EE. Our findings illustrated that the EE can be improved considerably through integration of CoMP-SWIPT technology in HetNets.

\section{REFERENCES}

[1] J. G. Andrews, "Seven ways that HetNets are a cellular paradigm shift," IEEE Commun. Mag., vol. 51, no. 3, pp. 136-144, Mar. 2013.

[2] M. K. Karakayali, G. J. Foschini, and R. A. Valenzuela, "Network coordination for spectrally efficient communications in cellular systems," IEEE Wireless Commun. Mag., vol. 13, no. 4, pp. 56 - 61, Aug. 2006.

[3] Q. Cui, H. Wang, P. Hu, X. Tao, P. Zhang, J. Hamalainen, and L. Xia, "Evolution of limited-feedback comp systems from 4G to 5G: CoMP features and limited-feedback approaches," IEEE Veh. Tech. Mag., vol. 9, no. 3, pp. 94 - 103, Sep. 2014.

[4] X. Lu, P. Wang, D. Niyato, D. I. Kim, and Z. Han, "Wireless networks with RF energy harvesting: A contemporary survey," Commun. Surveys Tuts., vol. 17, no. 2, pp. 757-789, 2nd Quart. 2015.

[5] N. Zhao, F. R. Yu, and V. C. M. Leung, "Wireless energy harvesting in interference alignment networks," IEEE Commun. Mag., vol. 53, no. 6, pp. $72-78$, June 2015.

[6] L. R. Varshney, "Transporting information and energy simultaneously," in Proc. 2008 IEEE Int. Symp. Inf. Theory, July 2008, pp. 1612-1616.

[7] D. W. K. Ng, E. Lo, and R. Schober, "Wireless information and power transfer: energy efficiency optimization in OFDMA systems," IEEE Trans. Wireless Commun., vol. 12, no. 12, pp. 6352-6370, Dec. 2013.

[8] J. Tang, D. K. C. So, A. Shojaeifard, K. K. Wong, and J. Wen, "Joint antenna selection and spatial switching for energy efficient MIMO SWIPT system," IEEE Trans. on Wireless Commun., vol. 16, no. 7, pp. 4754 - 4769, July 2017.

[9] Z. Xu, C. Yang, G. Y. Li, Y. Liu, and S. Xu, "Energy-efficient CoMP precoding in heterogeneous networks," IEEE Trans. Sig. Process., vol. 62, no. 4, pp. 1005 - 1017, Feb. 2014.

[10] W. Dinkelbach, "On nonlinear fractional programming," Management Science, vol. 13, pp. 492-498, Mar. 1967.

[11] S. Boyd and L. Vandenberghe, Convex Optimization. Cambridge University Press, Cambridge, UK, 2004.

[12] J. Tang, D. So, E. Alsusa, K. A. Hamdi, and A. Shojaeifard, "Resource allocation for energy efficiency optimization in heterogeneous networks," IEEE Journal on Sel. Areas in Commun., vol. 33, no. 10, pp. 21042117, Oct. 2015. 